\title{
Gunshot Injury of Pelvi-Ureteric Junction: Management by the Rendezvous Technique
}

\author{
Virender Sekhon, MS, MD, and Manav Suryavanshi, MS, MD²
}

\begin{abstract}
Background: Gunshot injury of ureter is common, but isolated injury of pelvi-ureteric junction (PUJ) has not been reported. Moreover, its management is evolving from the traditional urinary diversion, stenting followed later by definitive surgical repair to a more upfront minimally invasive endourologic approach.

Case Presentation: An adolescent boy presented with gunshot laceration injury of left PUJ with associated small intestinal injury. Radiological investigations confirmed a contained urinary leak. Retrograde stenting was not effective. The same guidewire was retained into the urinoma and an antegrade percutaneous access was obtained. A snare was used to retrieve the guidewire and obtain a through and through access. A ureteropelvic drainage catheter was inserted over this guidewire and secured in the upper ureter, bypassing the region of injury. Three weeks later, the drainage tube was removed and the same tract was used for antegrade stenting. A retrograde pyelogram done 3 months later during stent removal demonstrated no leak. The patient is asymptomatic for urinary symptoms on 6 months of follow-up.
\end{abstract}

Conclusion: Rendezvous technique is a feasible alternative to open repair of gunshot pelvi-ureteric junction injury. Long-term follow-up is awaited.

Keywords: kidney pelvis, laceration, mesenteric artery, superior, rendezvous technique, wounds, gunshot

\section{Introduction and Background}

$\mathbf{U}$

RINARY TRACT INVOLVEMENT Occurs in $3 \%$ to $10 \%$ of all traumatic injuries. ${ }^{1}$ Of these, gunshot wounds (GSWs) usually involve bladder, ureter and urethra. GSW penetrating the pelvi-ureteric junction (PUJ) is rare, with no elaborate mention in the literature. Ureteral injuries have traditionally been managed by diversion nephrostomy and stenting, followed by definitive surgical repair. ${ }^{2}$ It is plausible that a similar treatment algorithm may be followed for more proximal urinary tract injuries.

We discuss the feasibility of a combination of antegrade and retrograde image-guided techniques for management of gunshot PUJ injury in an adolescent.

\section{Presentation of Case}

A 16-year-old male presented with point-blank gunshot abdominal injury. Contrast enhanced computed tomography of the abdomen revealed gangrenous bowel because of superior mesenteric artery blowout along with laceration of the left PUJ with contained urinary leak (Fig. 1).

Poststabilization, emergency exploratory laparotomy was performed with resection of gangrenous bowel and exteriorization. During the same operative sitting, an attempt to retrogradely negotiate a $0.018^{\prime \prime}$ hydrophilic guidewire across the transected PUJ failed. Retrograde pyelogram demonstrated contrast leak at the level of PUJ into the retroperitoneal cavity. Retrograde ureterography confirmed similar findings. While retaining the guidewire into the urinoma, the

Sections of ${ }^{1}$ Paediatric Urology and ${ }^{2}$ Endourology, Division of Urology, Renal Transplant and Robotics, Medanta-The Medicity, Gurgaon, India.

(C) Virender Sekhon and Manav Suryavanshi 2017; Published by Mary Ann Liebert, Inc. This is an Open Access article distributed under the terms of the Creative Commons Attribution License, which permits unrestricted use, distribution, and reproduction in any medium, provided the original work is properly cited. 


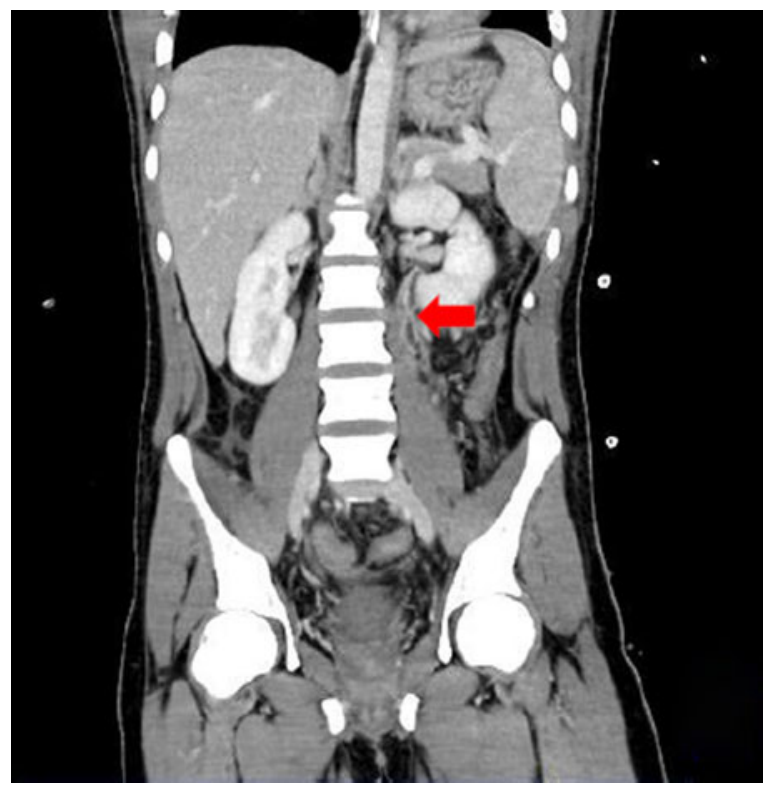

FIG. 1. Contrast-enhanced computed tomography of the abdomen showing contrast leak at the level of left pelviureteric junction (arrow).

patient was turned prone and an antegrade percutaneous access was obtained and leak was confirmed (Fig. 2a). The pelvi-ureteric defect appeared to be nearly $3 \mathrm{~cm}$ in length on imaging, with a thin contrast lining one wall of the PUJ. A gooseneck snare introduced through the access sheath was used to retrieve the guidewire from the percutaneous sheath to attain a complete through and through access (Fig. 2b). A 16F ureteropelvic drainage catheter was inserted over the guidewire to smoothly bypass the area of injury and was placed into the upper ureter for external drainage (Fig. 2c). The retroperitoneal urinoma resolved completely 3 weeks postdrainage. Thereafter, a $6 \mathrm{~F} 26 \mathrm{~cm}$ Double-J stent was placed in an antegrade manner using the same tract, and nephrostomy tube was removed. The stent was retained in situ for 12 weeks and a retrograde pyelogram during stent removal was negative for any leak or jet sign. The patient is presently asymptomatic under follow-up for 6 months poststent removal, with no hydronephrosis on ultrasonography and a prompt excretion across the PUJ on a renal dynamic scan with preserved renal function. He has been maintained on supportive parenteral nutrition for the short bowel and is awaiting intestinal transplant.

\section{Discussion and Review of Literature}

Pardilidis et al. have noted well that endourology, along with laparoscopy, has completely revolutionized the management of the upper tract. ${ }^{3}$ The underlying principle of management of laceration or transection injuries of the urinary tract is restoring the anatomical integrity. This can be achieved by retrograde, antegrade, or the more recently described combined approaches (rendezvous technique). ${ }^{4}$ The latter technique involves passage of a guidewire from one side to be retrieved from the opposite access site. This can be achieved by using a snare or a forceps under fluoroscopy or endoscopy through a nephroscope or ureteroscope. ${ }^{4}$ A similar technique was used in the index case. Utmost care has to be exercised during the maneuver to not convert the partial disruption into a complete transection.

There is abundant literature on GSWs to the ureter. PerezBrayfield and colleagues reviewed 118 ureteral injury patients from GSWs. ${ }^{5}$ Ureteral injuries are associated with other intraabdominal organ injuries in $85 \%$ of the cases. Small intestine is the most commonly associated organ like in the index case. Bullet injuries, even if single with a discernable trajectory, still need a high index of suspicion during intraoperative evaluation for identification of ureteral injuries. Most articles emphasize on aggressive surgical management with adequate debridement and spatulated, tension-free, splinted, water-tight anastomosis with diversion. Stricture at the site of injury is a plausible complication after the initial management. Hence, a long-term follow-up for these patients is suggested.

To the best of our knowledge, this is the first article describing gunshot injury to the PUJ along with blow out of the a

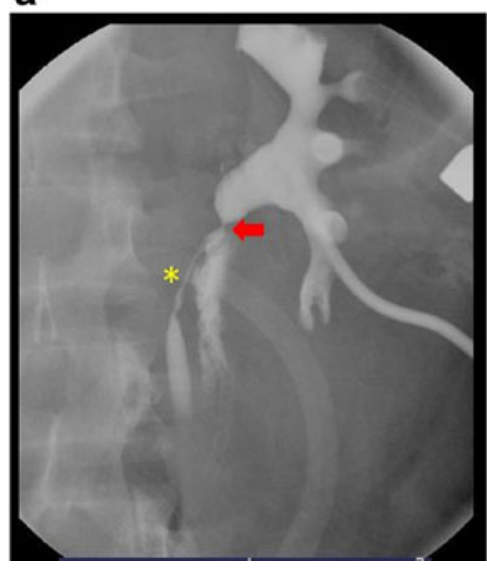

b

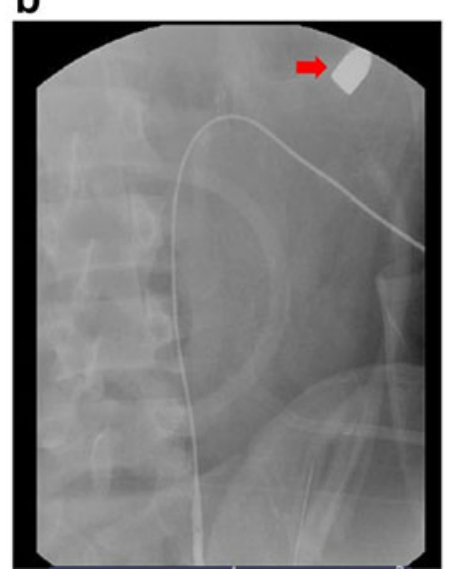

C

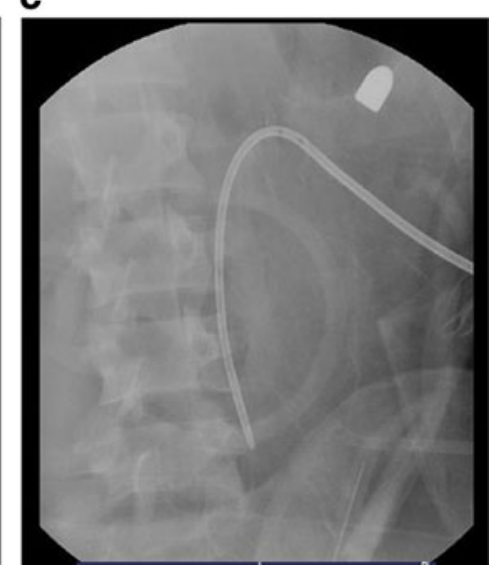

FIG. 2. (a) Antegrade nephrostogram showing contrast leakage at the left pelvi-ureteric junction (arrow); streak of contrast delineating the upper ureter (asterisk). (b) Through and through passage of guidewire using the rendezvous technique. (c) Ureteropelvic drainage catheter across the area of pelvi-ureretic junction transection and well into the ureter. 
superior mesenteric artery, managed by minimally invasive endourologic technique.

\section{Conclusion}

A combination of the antegrade and retrograde approaches or the rendezvous technique is a feasible alternative to open surgical repair of gunshot injury to PUJ. However, further studies with long-term follow-up are desirable.

\section{Disclosure Statement}

No competing financial interests exist.

\section{References}

1. Krieger JN, Algood CB, Mason JT, et al. Urological trauma in the Pacific Northwest: Etiology, distribution, management, and outcome. J Urol 1984;132:70-73.

2. Al-Ali M, Haddad LF. The late treatment of 63 overlooked or complicated ureteral missile injuries: The promise of nephrostomy and role of autotransplantation. J Urol 1996; 156:1918-1921.

3. Pardilidis NP, Papatsoris AG, Kosmaoglou EV. Endoscopic and laparoscopic treatment of ureteropelvic junction obstruction. J Urol 2002;168:1937-1940.

4. Arabi M, Mat'hami A, Said MT, et al. Image-guided ureteral reconstruction using rendezvous technique for complex ureteric transection after gunshot injuries. Avicenna J Med 2016;6:28-30.
5. Perez-Brayfield MR, Thomas E, Anand K, et al. Gunshot wounds to the ureter: A 40-year experience at Grady Memorial Hospital. J Urol 2001;1669:119-121.

Address correspondence to:

Virender Sekhon, MS, MD

Section of Paediatric Urology

Division of Urology, Renal Transplant and Robotics

Medanta-The Medicity

Gurgaon 122001

Haryana

India

E-mail: virender.sekhon@gmail.com

Cite this article as: Sekhon V, Suryavanshi M (2017)

Gunshot injury of pelvi-ureteric junction: management by the rendezvous technique, Journal of Endourology Case Reports 3:1, 78-80, DOI: 10.1089/cren.2017.0040. 